\title{
Optimal electromagnetic energy extraction from transverse galloping
}

\author{
D. Vicente-Ludlam, A. Barrero-Gil*, A. Velazquez \\ Aerospace Propulsion and Fluid Mechanics Department, School of Aeronautics, Universidad Politecnica de Madrid, Plaza Cardenal \\ Cisneros 3, E-28040 Madrid, Spain
}

\begin{abstract}
A B S T R A C T
A fully coupled electro-fluid-elastic model for electromagnetic energy harvesting from Transverse Galloping is presented here. The model considers a one degree-of-freedom galloping oscillator where fluid forces are described resorting to quasi-steady conditions; the electromagnetic generator is modelled by an equivalent electrical circuit where power is dissipated at an electrical load resistance; the galloping oscillator and the electromagnetic model are coupled appropriately. Two different levels of simplification have been made depending on the comparison between the characteristic electrical and mechanical timescales. The effect of the electrical resistance load on the energy harvested is studied theoretically. For fixed geometry and mechanical parameters, it has been found that there exists an optimal electrical resistance load for each reduced velocity. On the practical side, this result can be helpful to design tracking-point strategies to maximize energy harvesting for variable flow velocity conditions.
\end{abstract}

\section{Introduction}

In recent years, Flow-Induced Vibrations like Vortex-induced Vibrations (VIV), Transverse Galloping (TG) oscillations or Flutter have been considered as a new mean to harvest energy from fluid flows (Bernitsas et al., 2008; Barrero-Gil et al., 2010, 2012; Sanchez-Sanz et al., 2009; Grouthier et al., 2013; Abdelkefi et al., 2013; Doare and Michelin, 2011; Allen and Smits, 2001, to name only a few). The basic idea is to take advantage of these phenomena to convert part of the kinetic energy of the flow into oscillatory mechanical energy of the elastic body; thereafter, the mechanical energy of the body may be converted into electrical energy by electromagnetic, piezoelectric, or electrostatic means.

TG potential for energy harvesting has been recently investigated theoretically, numerically, and experimentally by Barrero-Gil et al. (2010), Abdelkefi and co-workers (2012, 2013), Sirohi and Mahadik (2011), or Zhao et al. (2012). Briefly outlined, it can be said that TG is a fluid-elastic instability that appears in some elastic bluff bodies when the velocity of the incident flow exceeds a critical value. Then, a small transverse displacement of the body induces an angle of attack relative to the incoming flow and a fluid force appears in the direction of the displacement in such a way that energy is pumped from the current to the body. Oscillatory motion (transverse to the flow) develops with an increasing amplitude until the energy dissipated per cycle by mechanical damping balances the energy input per cycle from the flow. If the geometry of the body and the elastic properties are appropriate, the instability may appear at low flow velocities and with large excitation

\footnotetext{
${ }^{*}$ Corresponding author.

E-mail address: antonio.barrero@upm.es (A. Barrero-Gil).
} 
amplitudes, making TG a very promising way to harvest energy successfully. For an authoritative introduction to TG phenomenology and modelling, the reader is referred to Parkinson (1989), or the books by Blevins (1990), Naudascher and Rockwell (1994), or Païdoussis et al. (2011).

Barrero-Gil et al. (2010) developed a theoretical model to study TG potential for energy harvesting. They considered a one degree of freedom model to describe galloping oscillations and a quasi-steady approximation to describe TG fluid forces. They found analytically the dependence of the energy transferred from the flow to the galloping body as a function of the main governing parameters, namely the mass ratio (i.e. the ratio of the mean density of the galloping body with respect to the density of the surrounding flow), the mechanical damping, the fluid flow velocity, and the geometry of the body. For the sake of simplicity, they just considered a generic viscous damper to dissipate the energy pumped from the flow. Here, a forward step is presented in order to model the TG dynamics when an electromagnetic generator is used to produce electrical current which is dissipated at a generic electrical load resistance. To this end, the model presented at Barrero-Gil et al. (2010) is now coupled with a mathematical model of the electromagnetic generator.

As will be shown in detail later, the electrical generator model may be simplified to a large extent, allowing an analytical solution, if the characteristic electrical timescale of the electromagnet generator $T_{E}$ is much shorter than the characteristic timescale of galloping oscillations $T_{N}\left(T_{E} \ll T_{N}\right)$. However, a perturbation approach has also been developed in order to gain deeper understanding of the dynamics when $\left(T_{E} / T_{N}\right)<1$ and the inductance of the electromagnetic generator plays a role. In both cases, the effect of the electrical resistance load on the energy harvested is studied theoretically. For fixed geometry of the galloping body and mechanical parameters, it has been found that there exists an optimal electrical resistance load that maximizes the energy harvested for each flow velocity. From the practical side, this result can be helpful when designing some tracking-point strategy to maximize energy harvesting for changing flow velocity conditions.

First of all, in Section 2, an electro-fluid-elastic model is introduced to describe the coupling between the galloping body and the electromagnetic generator dynamics. Section 3.1 presents and discusses analytical results for the most simplified situation $\left(T_{E} \ll T_{N}\right)$, whereas Section 3.2 is devoted to studying the case when the disparity of electrical and mechanical timescales is not so large but $\left(T_{E} / T_{N}\right)<1$. In this case, a perturbation approach is introduced to make an analytical treatment. Finally, Section 4 presents some concluding remarks.

\section{Mathematical model}

Let us consider a simplified dynamical system which consists of a spring-mounted prismatic body, prone to galloping, in an incoming flow. The assembly can be better understood in Fig. 1(a). As it can be seen, the system is composed of a viscous parasite damper, with constant damping per unit length $c$, a linear spring, with constant stiffness per unit length $k$, and the electromagnetic generator used as energy harvester, where the magnet is linked to the prism, so it oscillates relative to the coil producing electricity which is dissipated in an electrical resistive load $R_{L}$. The prism has a mass per unit length of $m$ and is restricted to move in the $y$ direction (transverse to the incident flow).

Appropriate balance between inertia, damping, stiffness, electromechanical, and fluid forces in the system gives the following ordinary differential equation:

$$
m \ddot{y}+c \dot{y}+k y=\frac{1}{2} \rho U^{2} D C_{Y}-F_{F E M},
$$

(a)

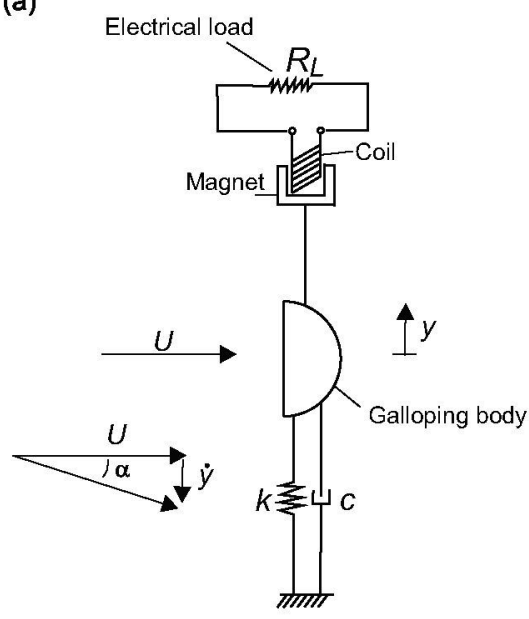

(b)

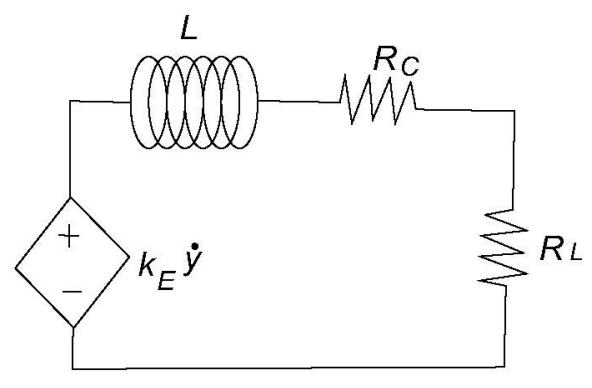

Fig. 1. (a) Sketch of the spring-mounted galloping body in cross-flow and linked to an electromagnetic generator. (b) Equivalent electrical circuit of the electromagnetic generator. 
where $y$ denotes the transverse position of the prism, $\rho$ is the fluid density, $U$ is the undisturbed velocity of the incident flow, $D$ is the characteristic dimension of the prism prone to gallop normal to the flow, $C_{Y}$ is the instantaneous fluid force coefficient in the transverse direction to the incident flow, $F_{F F M}$ is the electromagnetic force per unit length in the $y$ direction and, finally, the dot symbol stands for differentiation with respect to physical time $t$. Note that for the sake of simplicity, damping and stiffness forces have been considered to be linear. For an introduction about how non-linear effects can be introduced and mathematically modelled the reader is referred to Lee et al. (2011).

In order to describe $C_{Y}$, the quasi-steady hypothesis is resorted to, since it is assumed that the characteristic timescale of the prism oscillation (of order $2 \pi(m / k)^{1 / 2}$ ) is much larger than the characteristic timescale of the flow (of order $D / U$ ). Then, the fluid force is only dependent on the instantaneous attitude of the prism with respect to the flow, which can be described by the motion-induced angle of attack $\alpha$. Since the motion-induced angle of attack is given by $\alpha=\tan ^{-1}(\dot{y} / U)$, the fluid force can be introduced as an empirical function of $\dot{y}$, which can be approximated by a polynomial when the static variation of $C_{Y}$ with $\tan \alpha$ is known (Blevins, 1990). Observe that $C_{Y}$ can be related to the lift $C_{L}$ and drag $C_{D}$ coefficients since $C_{Y}=-\left(C_{L}+C_{D} \tan \alpha\right) / \cos \alpha$. For our purposes a cubic polynomial can be considered accurate enough to approximate the vertical fluid force coefficient:

$$
C_{Y}=a_{1} \frac{\dot{y}}{U}+a_{3}\left(\frac{\dot{y}}{U}\right)^{3},
$$

where $a_{1}$ and $a_{3}$ are the empirical coefficients to fit by a polynomial the $C_{Y}$ versus $\tan (\alpha)$ dependence measured in static tests. $a_{1}$ and $a_{3}$ are dependent mainly on the cross-section geometry of the prism. The linear coefficient $a_{1}$ is the slope of the vertical fluid force coefficient at zero angle of attack, and therefore it is necessary for it to be $a_{1}>0$ in order to obtain a galloping response (that is, linearized fluid forces act as a negative damping term when $a_{1}>0$ ). $a_{3}$ accounts for the nonlinear effects and it has to be negative.

Let us consider an equivalent electrical model to modelize the electromagnetic generator (see Fig. 1(b); El-hami et al., 2001), which gives a relationship between the main parameters of the electromagnet generator and the induced electromagnetic force $F_{F E M}$ :

$$
\begin{aligned}
& \left(R_{L}+R_{C}\right) i+L \frac{d i}{d t}=k_{E} \frac{d y}{d t}, \\
& F_{F E M}=k_{E} i,
\end{aligned}
$$

where $R_{L}$ is the resistance of the load connected to the generator, $R_{C}$ is the internal resistance of the coil, $L$ is its inductance, $k_{E}$ is an electromechanical coupling constant determined by the flux density of the magnetic field and the number of turns and length of the coil, and $i$ is the intensity circulating through the coil. Eqs. (3a) and (3b) constitute a simplified model of an electromagnet generator such as the one described in Rizzoni and Hartley (2000). Introducing dimensionless variables $Y=y / D$, $I=i / I_{C}$ and $\tau=\omega_{N} t$, with $\omega_{N}=(k / m)^{1 / 2}$ and $I_{C}=m \omega_{N}^{2} D / k_{E}$, one may rewrite Eqs. (1)-(3) in dimensionless form:

$$
\begin{aligned}
& Y^{\prime \prime}+2 \zeta Y^{\prime}+Y=\frac{U^{* 2}}{2 m^{*}}\left(a_{1} \frac{Y^{\prime}}{U^{*}}+a_{3}\left(\frac{Y^{\prime}}{U^{*}}\right)^{3}\right)-I, \\
& \beta I^{\prime}+I=2 \zeta_{E} Y^{\prime},
\end{aligned}
$$

where prime denotes the differentiation with respect to the dimensionless time, $\beta=\left(L \omega_{N}\right) /\left(R_{L}+R_{C}\right)$ is a relationship between the characteristic electrical and mechanical time, $\zeta=c /\left(2 m \omega_{N}\right)$ is the dimensionless parasite mechanical damping, $U^{*}=U /\left(\omega_{N} D\right)$ is the reduced velocity, $m^{*}=m /\left(\rho D^{2}\right)$ is the mass ratio and $\zeta_{E}$ is a dimensionless damping coefficient which is a result of the coupling of the dynamics of the prism and the electromagnetic generator:

$$
\zeta_{E}=\frac{k_{E}^{2}}{2 m \omega_{N}\left(R_{L}+R_{C}\right)}
$$

A conversion factor (or efficiency) can be defined as the mean power $P_{E}$ dissipated at the electrical load divided by the total power in the flow per unit length as

$$
\eta_{E}=\frac{P_{E}}{\frac{1}{2} \rho U^{3} D},
$$

where

$$
P_{E}=\frac{1}{T} \int_{0}^{T} R_{L} i^{2} d t
$$

The efficiency, as described in Eq. (6), is presented in the same way as in Barrero-Gil et al. (2010), or Grouthier et al. (2013), where the power harvested is compared to the power available in the flow in a section equivalent to the diameter of the prism. However, other ways of defining such an efficiency are possible. For instance, the efficiency can be defined with the power harvested compared to the power available in the flow in a section equivalent to the space swept by the prism during its oscillations. Both efficiencies might yield different results of optimal configuration as one stresses the importance of the 
maximum power extracted while the other one stresses the importance of maximizing the energy harvested per unit length in the transverse direction.

The system given by Eqs. (4) to (7) allows us to obtain the galloping response and the power dissipated at $R_{L}$ as a function of the mechanical parameters $\left(m^{*}, \zeta, \omega_{N}\right)$, geometrical and flow parameters $\left(U^{*}, a_{1}, a_{3}, D\right)$, and parameters of the electromagnet generator $\left(k_{E}, \beta, R_{C}\right)$. The system can be solved numerically and an optimization method might be used to obtain the optimal value of $R_{L}$ that maximizes the energy harvested for fixed values of other governing parameters. However, because of the large number of governing parameter this task may be costly in time and may not be so easy to establish design rules for $R_{L}$. In this sense, an analytical approach, which is presented in the following section, can be quite useful.

\section{Galloping response, energy efficiency, and optimal electrical load resistance}

\subsection{Low frequency oscillations. $\beta \ll 1$}

If $\beta \ll 1$ is considered, it means that the effect of the inductance on the dynamic response is negligible and in this case, an easy simplification can be considered, that is $i=2 \zeta_{E} I_{c} Y^{\prime}$ (see Eq. (4b)), and Eq. (4a) is then

$$
Y^{\prime \prime}+2 \zeta Y^{\prime}+Y=\frac{U^{* 2}}{2 m^{*}}\left(a_{1} \frac{Y^{\prime}}{U^{*}}+a_{3}\left(\frac{Y^{\prime}}{U^{*}}\right)^{3}\right)-2 \zeta_{E} Y^{\prime}
$$

Eq. (8) can be solved approximately when the nonlinearity of the fluid forcing term is weak compared to the linear forces (for $m^{*} U^{*} \geqslant 1$, which is usual when the fluid in consideration is air). In that case, one may assume that the steady movement response is sinusoidal, $Y=A^{*} \sin \left(\omega^{*} \tau\right)$, where $\omega^{*}=\omega / \omega_{N}$. Substituting this into Eq. (8), taking into account that $\cos ^{3}(\omega \tau) \approx 3 \cos (\omega \tau) / 4$, (i.e. higher order terms in $\cos \left(3 \omega^{*} \tau\right)$ are neglected) which is valid when the system is narrowband in frequency such as for the case in study, and equating sine and cosine terms, after some algebra one gets

$$
\begin{aligned}
& A^{*}=\left(\frac{4 U^{*}}{3 a_{3}}\left(4 m^{*} \zeta_{T}-a_{1} U^{*}\right)\right)^{1 / 2}, \\
& \omega^{*}=1,
\end{aligned}
$$

where $\zeta_{T}=\zeta+\zeta_{E}$. Observe that galloping oscillations $\left(A^{*}>0\right)$ are only possible when $U^{*}>U_{g}^{*}=4 m^{*} \zeta_{T} / a_{1}$. It is important to remark that the value of $U_{g}^{*}$ depends on the load resistance $R_{L}$ (via $\zeta_{E}$ ), which is a primary result of the ongoing study.

The mean power per unit length is (see Eq. (7))

$$
P_{E}=\frac{4}{2} R_{L} \zeta_{E}^{2} I_{C}^{2} A^{* 2} \omega^{* 2}=\frac{8 R_{L} \zeta_{E}^{2} I_{C}^{2} U^{*}}{3 a_{3}}\left(4 m^{*} \zeta_{T}-a_{1} U^{*}\right),
$$

and the efficiency

$$
\eta_{E}=\frac{16 R_{L} \zeta_{E}^{2} I_{C}^{2}}{3 a_{3} \rho \omega_{n}^{3} D^{4} U^{* 2}}\left(4 m^{*} \zeta_{T}-a_{1} U^{*}\right) .
$$

Eq. (11) can be rewritten in order to express all variables non-dimensionally as

$$
\eta_{E}=\frac{8 m^{*} \zeta_{E}}{3 a_{3}\left(1+R_{C} / R_{L}\right) U^{* 2}}\left(4 m^{*} \zeta_{T}-a_{1} U^{*}\right) .
$$

\subsubsection{Maximum efficiency achievable for fixed $R_{L}$}

The conversion factor (or efficiency) is a function of $R_{L}$ and $U^{*}$, so in order to obtain the maximum efficiency, it is necessary to obtain the partial derivatives of Eq. (12) with respect to $U^{*}$ and $R_{L}$, equate to zero and solve the system of 2 equations and 2 unknowns:

$$
\begin{aligned}
\frac{\partial \eta_{E}}{\partial U^{*}} & =0, \\
\frac{\partial \eta_{E}}{\partial R_{L}} & =0 .
\end{aligned}
$$

In first place, if $R_{L}$ is considered to be a fixed value, then only Eq. (13a) applies. By solving Eq. (13a) it is possible to retrieve the reduced velocity at which the maximum efficiency is achieved, giving a value of reduced velocity of $2 U_{g}^{*}$ :

$$
U_{\eta_{E}^{\max }}^{*}=2 U_{g}^{*}=\frac{8 m^{*}\left(\zeta+\zeta_{E}\right)}{a_{1}}
$$


Substitution of Eq. (14) into Eq. (12) allows us to get the maximum efficiency achievable when $R_{L}$ is fixed, given by

$$
\eta_{E}^{\max }=-\frac{a_{1}^{2}}{6 a_{3}}\left(\frac{1}{1+R_{C} / R_{L}}\right)\left(\frac{1}{1+\zeta / \zeta_{E}}\right),
$$

which relates the maximum achievable efficiency with the cross-section geometry of the prism $\left(a_{1}\right.$ and $\left.a_{3}\right), R_{C} / R_{L}$, and the ratio between the parasite damping and the electrical damping $\zeta / \zeta_{E}$.

A universal curve for the efficiency can be introduced using two new normalized variables, $\tilde{\eta}=\eta_{E} / \eta_{E}^{\max }$ and $\tilde{U}=U^{*} / U_{g}^{*}$ and substituting them into Eq. (12)

$$
\tilde{\eta}_{E}=-\frac{4(1-\tilde{U})}{\tilde{U}^{2}}
$$

With these re-normalized variables, it is possible to collapse the efficiency curves for all electrical/mechanical configurations into a single curve (Eq. (16)) providing information about the transverse galloping response over a range of reduced incident velocities. From Fig. 2, once galloping is started $(\tilde{U}=1)$, the normalized efficiency increases rapidly with the normalized reduced velocity until a value of $\tilde{U}=2$ is reached obtaining a value of the normalized efficiency of $\tilde{\eta}=1$, then the normalized efficiency diminishes slowly with $\tilde{U}$.

Thus, it is important to correctly tune the value of $R_{L}$ so as to modify $U_{g}^{*}$ and correspondingly be at the maximum possible efficiency for each value of the reduced velocity and obtaining a broadband type of response.

\subsubsection{Optimal electrical load resistance for maximum power transfer at any instant}

From the point of view of designing a control law capable of harnessing the maximum possible power at any instant (similar to a tracking-point strategy for changing flow velocity conditions), $R_{L}$ has to be consequently modified depending on $U^{*}$. In this case, it is only necessary to solve Eq. (13b) and by doing so, it is possible to obtain the electric load that maximizes the power harvested for each value of the reduced velocity:

$$
R_{\text {Lop }}=\frac{p}{q}+\left(\left(\frac{p}{q}\right)^{2}+R_{C}\left(R_{C}-\frac{p}{q}\right)\right)^{1 / 2},
$$

where $p=4 m^{*} k_{E}^{2} /\left(2 m \omega_{N}\right)$ and $q=a_{1} U^{*}-4 m^{*} \zeta$.

Eq. (17) gives the optimal load electrical resistance as a function of the reduced velocity, geometry of the galloping body $\left(a_{1}\right)$, the mechanical parameters of the galloping system $\left(m, m^{*}, \zeta\right.$, and $\left.\omega_{N}\right)$, and electric properties $k_{E}$ and $R_{C}$ of the electromagnet. When $R_{L}$ is large compared to $R_{C}$, which should be a usual condition from the practical point of view, Eq. (17) is further simplified and $R_{\text {Lop }}$ approximates to

$$
R_{\text {Lop }}=\frac{2 p}{q}=\frac{4 m^{*} k_{E}^{2}}{m \omega_{N}\left(a_{1} U^{*}-4 m^{*} \zeta\right)}
$$

Observe that this equation suggests that $R_{L o p}$ scales as $U^{*-1}$ for large values of $U^{*}$.

If the resistance $R_{L}$ is tuned as suggested at Eq. (17) for each reduced velocity, then the maximum possible power is being transferred from the flow to the electrical load. Regarding the issue of power transfer to the electrical generator, it is in order

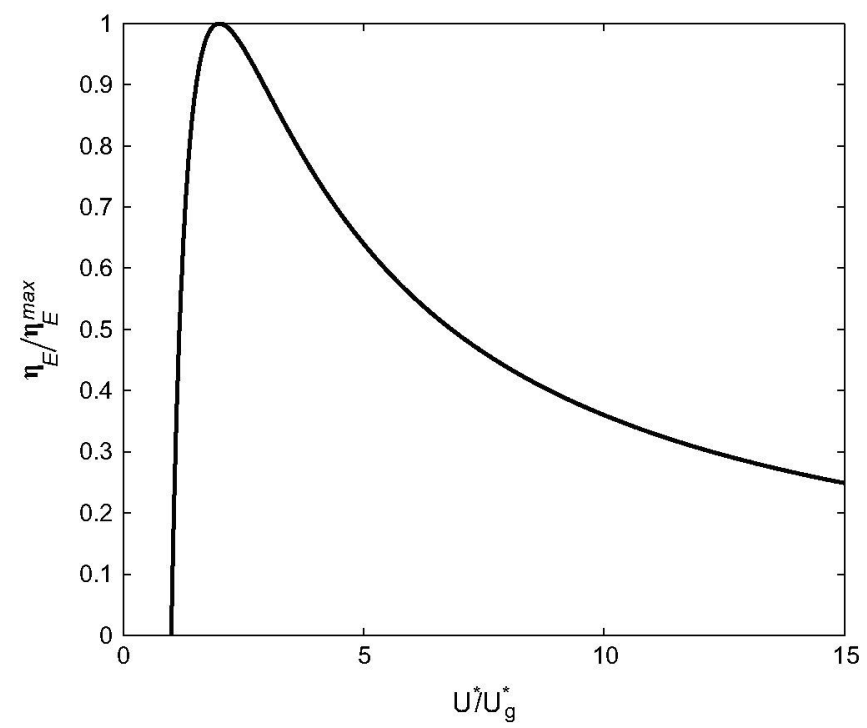

Fig. 2. Universal plot of the efficiency versus the flow velocity. 
to refer, in broad terms, to the Maximum Power Transfer Theorem. This theorem states that for a given constant voltage supply, the maximum power transferred to the electrical load $R_{L}$ occurs whenever $R_{L}=R_{C}$. In practice, this theorem suggests a way to solve the actual engineering problem of tuning the generator parameters so as to optimize power transfer. However, in the case presented in this paper, the Maximum Power Transfer Theorem cannot be applied straightforwardly because the supply voltage is not constant. In particular, voltage behavior is sinusoidal and it depends on both the reduced velocity and $R_{L}$ itself. Thereby, actual tuning of the generator parameters requires solving Eq. (13b) as previously stated. Nevertheless, it is to be noted that in the asymptotic limit of very large reduced velocity the voltage becomes less and less dependent on load resistance, see Eq. (10), and therefore, in this distinguished limit, the relation provided by the classical Maximum Power Transfer Theorem is recovered.

From the practical side, the variation of the load resistance could be done using a DC/DC converter capable of varying the equivalent electric load the galloping prism sees in order to always capture the maximum energy for the whole range of reduced velocities. This is a usual application in wind turbines with battery charging applications such as De Broe et al. (1999) and Moor and Beukes (2004). A Maximum Power Point Tracking (MPPT) system can also be used. This system can be fed with a look-up table with the actuation map as obtained in Eq. (17) or by using algorithms which do not use any prior information and by using a control system they automatically obtain the optimal load resistance as proposed in Koutroulis and Kalaitzakis (2006).

Finally, there is a maximum absolute efficiency point which fulfills both Eqs. (13a) and (13b) given by

$$
R_{\text {Imax }}=\sqrt{R_{C}\left(R_{C}+\frac{p}{4 m * \zeta}\right)},
$$

which is independent of $a_{1}$ and $a_{3}$. Once the value of $R_{I \max }$ that maximizes the efficiency is known, the value of the reduced velocity at which such maximum happens is also determined:

$$
U_{\max }^{*}\left(R_{L \max }\right)=2 U_{g}^{*}\left(R_{L \max }\right)=\frac{8 m^{*}\left(\zeta+\zeta_{E}\left(R_{L \max }\right)\right)}{a_{1}},
$$

and

$$
\eta_{E}^{a b s, \max }=-\frac{a_{1}^{2}}{6 a_{3}}\left(\frac{1}{1+R_{C} / R_{L \max }}\right)\left(\frac{1}{1+\zeta / \zeta_{E}\left(R_{L \max }\right)}\right) .
$$

\subsubsection{Practical example}

It may be now of interest to discuss a practical example that illustrates the theoretical results obtained previously. It consists in a spring-mounted prism with a D-type cross-section which is linked to the magnet of an electromagnet in such a way that the magnet can oscillate relative to the coil; the prism is under the action of an airstream and the electromagnet has a maximum rated power of $50 \mathrm{~W}$. Physical properties of the galloping body and the electromagnet generator are listed in Table 1.

Fig. 3(a) shows the optimal load electrical resistance $R_{\text {Lop }}$ that should be connected at the electromagnet at each wind speed (computed from Eq. (17)). Note how $R_{\text {Lop }}$ diminishes as wind speed increases; note also that $R_{\text {Lop }}$ is very large with respect to $R_{C}$ (for $U^{*}=50, R_{L o p}$ is still 24 times larger than $R_{C}$ ). Fig. 3(b) shows the electrical power variation with the wind speed when the load electrical resistance is optimally tuned and Fig. 3(c) shows the efficiency behavior with the reduced velocity (wind speed) when the load electrical resistance is optimally tuned; observe that because of non-zero mechanical parasite damping (remember that $\zeta=0.002$ ) and electrical losses at $R_{C}$, the maximum efficiency has diminished from the ideal value of $0.54\left(-a_{1}^{2} /\left(6 a_{3}\right)\right)$ down to 0.51 ; this absolute maximum value has been achieved with $R_{\operatorname{Lmax}}=420 \Omega$ at

Table 1

Physical properties of galloping body and the electromagnet generator. Values of the coefficients $a_{1}$ and $a_{3}$ are taken from Barrero-Gil et al. (2010).

\begin{tabular}{ll}
\hline Variable & Value \\
\hline$R_{C}$ & $12.2 \mathrm{Ohms}$ \\
$k_{E}$ & $10.6 \mathrm{~N} / \mathrm{A}$ \\
$L$ & $0.0096 \mathrm{H}$ \\
$m$ & $0.62 \mathrm{~kg} / \mathrm{m}$ \\
$\zeta$ & 0.002 \\
$k$ & $6.2 \mathrm{~N} / \mathrm{m}$ \\
$a_{1}$ & 0.79 \\
$a_{3}$ & -0.19 \\
$\mathrm{D}$ & $0.1 \mathrm{~m}$ \\
$\mathrm{~L}$ & $0.5 \mathrm{~m}$ \\
$\rho$ & $1.25 \mathrm{~kg} / \mathrm{m}^{3}$ \\
$m^{*}$ & 50 \\
\hline
\end{tabular}


(a)

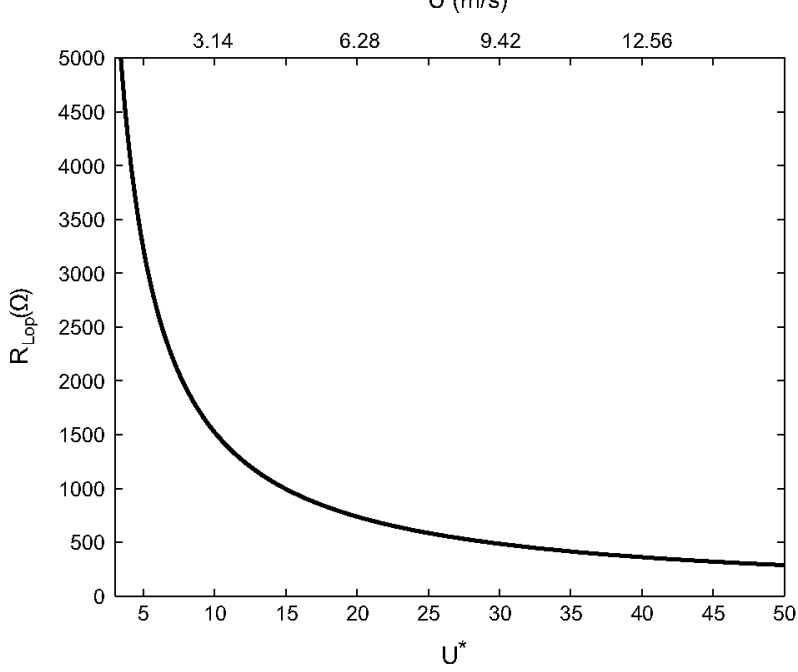

(c)

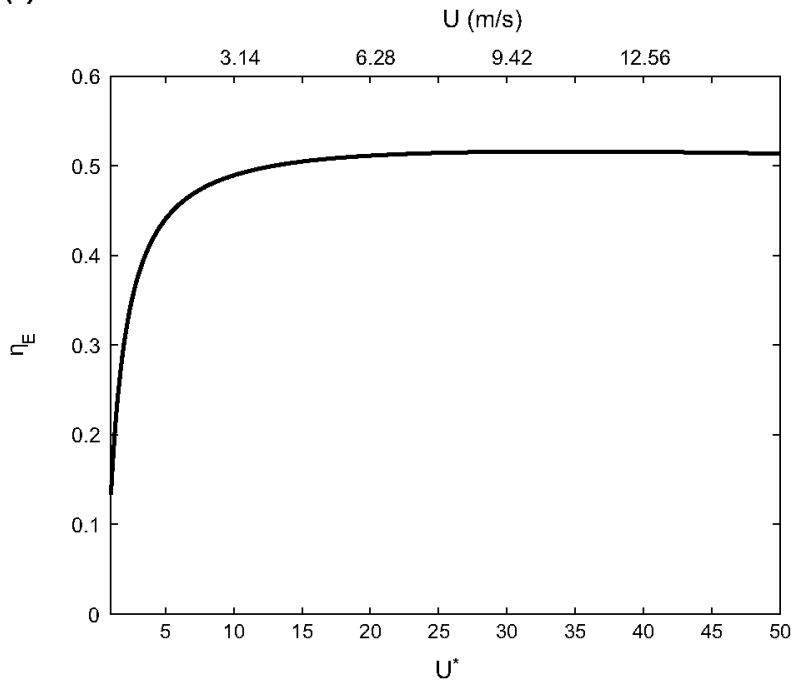

(b)

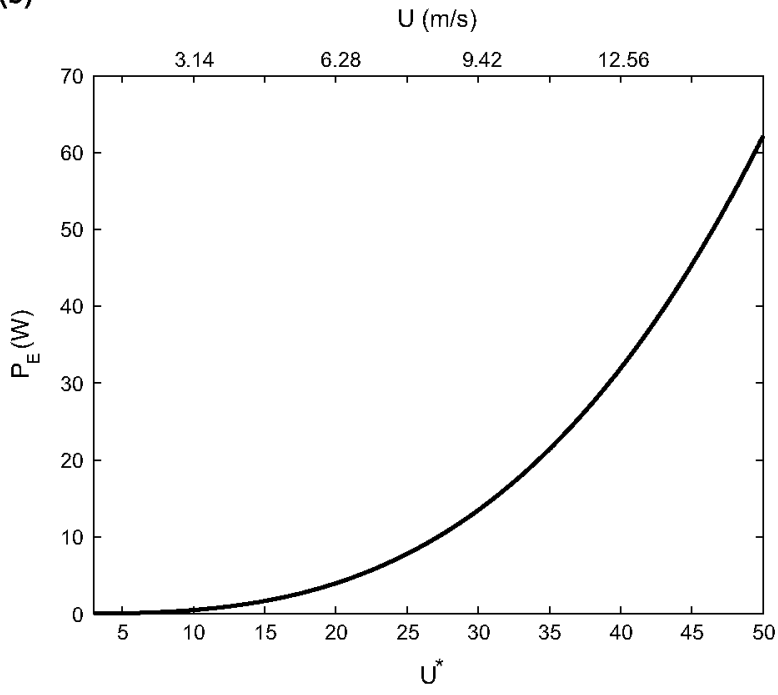

(d)

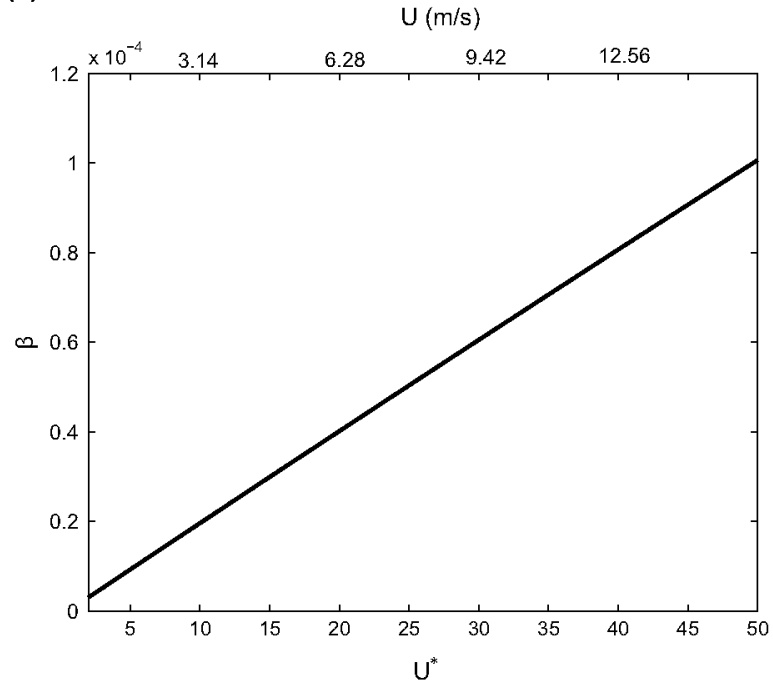

Fig. 3. Variation with the reduced velocity $U^{*}$ of the optimal electrical load resistance (a), output electrical power (b), efficiency (c), and $\beta$ (d).

$U_{\max }^{*}=34$ as predicted by Eqs. (19) and (20). Because of the optimal choice of $R_{L o p}$ for each wind speed, the efficiency dependence with the reduced velocity is diminished significantly and a broadband-type behavior can be observed. The idea is that, as $U^{*}$ increases, the electrical load resistance is diminished according to Eq. (17) in order to increase the electrical damping and thus increase $U_{\mathrm{g}}^{*}$ in such a way that $U^{*}$ is close to $2 U_{\mathrm{g}}^{*}$ at any instant, making possible to maintain the efficiency at high level for a larger range of reduced velocities. From the practical side this is a remarkable result since it is possible to get a low critical velocity of galloping and, at the same time, get high efficiency in a large range of reduced velocities. Observe that the potential to enlarge the range where efficiency is maintained at a high level is limited by $R_{C}$, since it imposes an upper limit for the electrical damping. Finally Fig. 3(d) shows the value of $\beta$ variation with reduced velocity in order to check that $\beta$ is always much lower than 1 .

Fig. 4(a) and (b) highlights the advantage associated with optimally choosing $R_{L}$ at any value of the reduced velocity. Fig. 4(a) shows the efficiency dependence with the reduced velocity when the electrical load resistance is optimal at any reduced velocity, as well as the efficiency dependence for two cases where the electrical load resistance takes a constant value. When $R_{L}$ takes a constant value, as the reduced velocity increases the efficiency increases until it reaches a maximum value (dependent on $R_{L}$ ). Further increase in $U^{*}$ results in a decrease in the efficiency which approaches to zero, so that the efficiency of energy conversion is in high levels only in a small range of reduced velocities: at low reduced velocities for large $R_{L}$ values and high reduced velocities for small $R_{L}$ values. However, when $R_{L}$ changes optimally with the reduced velocity, the efficiency increases with $U^{*}$ until it reaches a final value of high efficiency and then remains tenaciously at high levels almost 
(a)

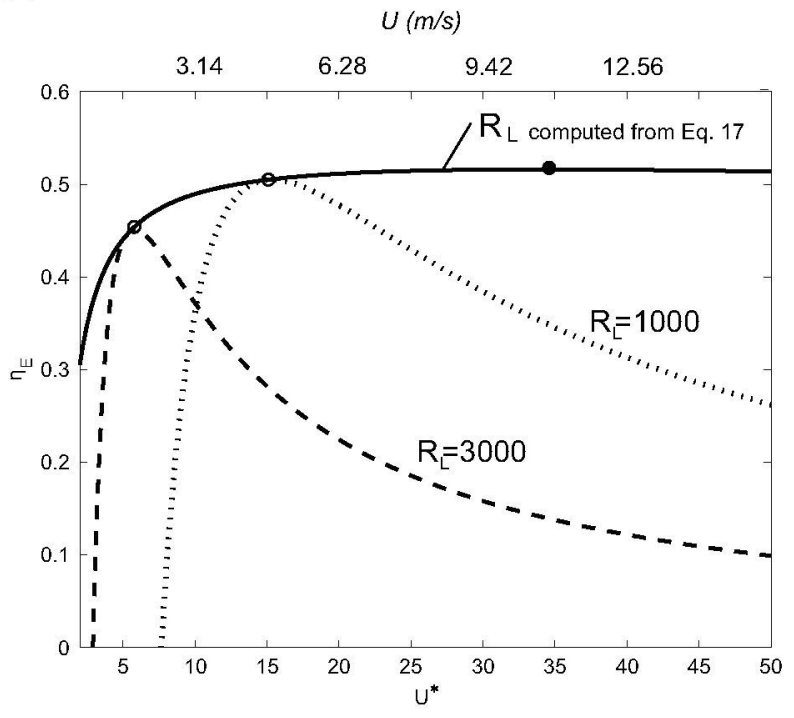

(b)

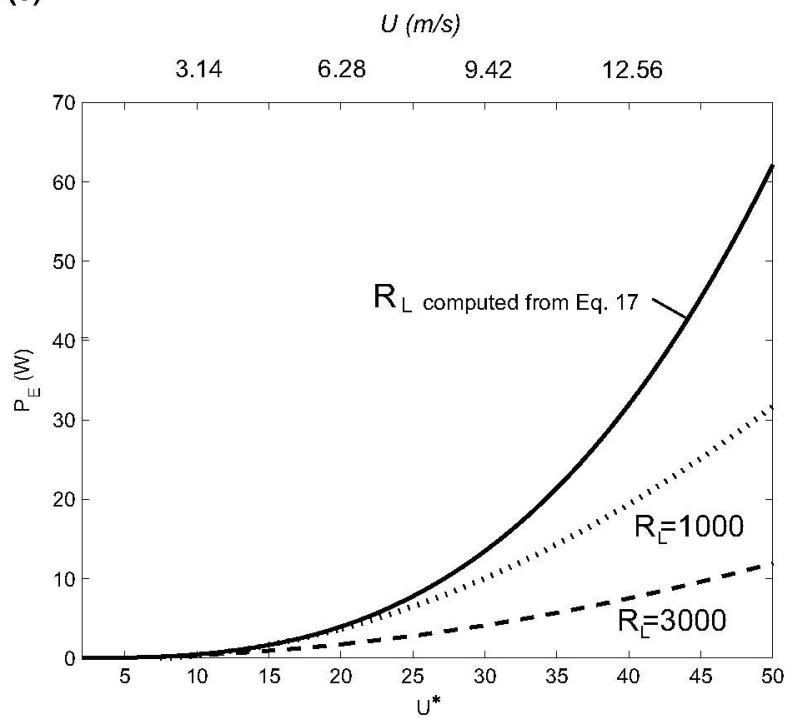

Fig. 4. (a) Efficiency variation with reduced velocity for optimal electrical load resistance (solid line) and for two different fixed values of the electrical load resistance (dashed lines). White circles denote the local maximum efficiency for a given $R_{L}$ whereas black circle denotes the absolute maximum efficiency. Note that the efficiency variation with reduced velocity for optimal electrical resistance (solid line) is the envelope of all local maximum efficiency points. (b) Output power variation with reduced velocity for optimal electrical load resistance (solid line) and for two different fixed values of the electrical load resistance (dotted line).

independent of the reduced velocity, which shows the advantages of adjusting continually and optimally the electrical resistance. In addition, when $R_{L}$ is optimally chosen the reduced velocity at which galloping starts (and power output begins) is low, but when $R_{I}$ takes a fixed value galloping oscillations can start at large reduced velocities (observe in Fig. 3(a) that efficiency curve starts around $U^{*}=3$ for $R_{L}=3000$ and $U^{*}=7.5$ for $R_{L}=1000$ ). Finally, it is apparent from Fig. 4(a) that, as discussed earlier, a re-normalization exits and all curves for $R_{L}$ constant collapse into a single one when redrawn in normalized variables $\tilde{U}$ and $\tilde{\eta}$.

In terms of electrical power output, the advantage of adjusting continually the electrical resistance $R_{L}$ with the reduced velocity over-taking a fixed value is also clear, as is can be seen in Fig. 4(b).

The energy harvesting efficiency response obtained can be compared to other devices. For example, in Abdelkefi et al. (2013), the same galloping model as presented here was taken into account, but the coupled electrical model used was a piezoelectric one. Results of efficiency for piezoelectric coupling leads to values much lower than the ones presented in this paper, between 0.01 and 0.03 . An experimental validation of electromagnetic energy extraction using wake galloping (not transverse galloping) through wind tunnel testing was carried out by Jung and Woo-Lee (2011); they measured efficiencies up to 0.094. In the VIVACE concept, the energy is generated via electromagnetic conversion and taking advantage from Vortex Induced Vibrations of a circular cylinder; efficiencies up to 0.31 were reported (see Raghavan, 2007) by correctly tuning the load resistance. While VIV is a resonance-type phenomenon where energy can only be harvested from the current for incident reduced velocities close to the resonance one, galloping has a much more broad type of response and by continuously changing $R_{\mathrm{I}}$, it is possible to even maintain high efficiency values for broader values of the reduced velocities.

\subsection{Moderate frequency oscillations $\beta<1$}

In some situations, requirements needed for $\beta \ll 1$ may be not fulfilled, so it is of interest to expand the domain of the analysis in order to look for the inductance effect when $\beta$ cannot be neglected and $\beta<1$. To this end, a perturbation expansion in $\beta$ is considered for the intensity in the electromagnet, $I=I_{0}+\beta I_{1}+\beta^{2} I_{2}$, in such a way that Eq. (4(b)) may be rewritten as

$$
\beta\left(I_{0}^{\prime}+\beta I_{1}^{\prime}+\beta^{2} I_{2}^{\prime}\right)+\left(I_{0}+\beta I_{1}+\beta^{2} I_{2}\right)=2 \zeta_{E} Y^{\prime} .
$$

Collecting, respectively, zero order terms, $\beta$ and $\beta^{2}$ order terms

$$
\begin{aligned}
& I_{0}=2 \zeta_{E} Y^{\prime}, \\
& I_{1}+I_{0}^{\prime}=0, \\
& I_{2}+I_{1}^{\prime}=0,
\end{aligned}
$$


and solving one after the other, one may get

$$
I=2 \zeta_{E}\left(Y^{\prime}-\beta Y^{\prime \prime}+\beta^{2} Y^{\prime \prime}\right)
$$

Substituting now Eq. (24) into Eq. (4a)

$$
Y^{\prime \prime}+2 \zeta Y^{\prime}+Y=\frac{U^{* 2}}{2 m^{*}}\left(a_{1} \frac{Y^{\prime}}{U^{*}}+a_{3}\left(\frac{Y^{\prime}}{U^{*}}\right)^{3}\right)-2 \zeta_{E} Y^{\prime}+2 \zeta_{E} \beta Y^{\prime \prime}-2 \zeta_{E} \beta^{2} Y^{\prime \prime \prime}
$$

The effect of the electrical inductance $(\beta)$ is to introduce a negative added mass (proportional to $\beta$ ) term as well as a negative damping term (proportional to $\beta^{2}$ ) into the dynamics of the galloping prism. Operating in the same way as previously, Eq. (25) can be analytically approximated when $m^{*} U^{*}$ is large enough. Assuming that the steady motion response is sinusoidal, $Y=A^{*} \sin \left(\omega^{*} t\right)$, where $\omega^{*}=\omega / \omega_{N}$, and substituting this into Eq. (25), after equating sine and cosine terms, and taking into account that $\cos ^{3}(\omega \tau) \approx 3 \cos (\omega \tau) / 4$ one gets

$$
\begin{aligned}
& A^{*}=\left(\frac{4 U^{*}\left(1-2 \zeta_{E} \beta\right)}{3 a_{3}}\left(4 m^{*} \zeta_{T}-a_{1} U^{*}-2 m^{*} \beta\left(\frac{2 \zeta_{E} \beta}{1-2 \zeta_{E} \beta}\right)\right)\right)^{1 / 2}, \\
& \omega^{* 2}=\frac{1}{1-2 \zeta_{E} \beta} .
\end{aligned}
$$

Since electrical power is proportional to $A^{* 2} \omega^{* 2}$ (see Eq. (10)), let us to collect $A^{*}$ and $\omega^{*}$ :

$$
A^{* 2} \omega^{* 2}=\left(\frac{4 U^{*}}{3 a_{3}}\left(4 m^{*} \zeta_{T}-a_{1} U^{*}-2 m^{*} \beta\left(\frac{2 \zeta_{E} \beta}{1-2 \zeta_{E} \beta}\right)\right)\right),
$$

which can be simplified if terms or higher order than $\beta^{2}$ are neglected

$$
A^{* 2} \omega^{* 2}=\left(\frac{4 U^{*}}{3 a_{3}}\left(4 m^{*} \zeta_{T}-a_{1} U^{*}-4 m^{*} \zeta_{E} \beta^{2}\right)\right)+O\left(\beta^{3}\right),
$$

since

$$
\beta \frac{2 \zeta_{E} \beta}{1-2 \zeta_{E} \beta}=2 \zeta_{E} \beta^{2}+O(\beta)^{3}
$$

Substituting Eq. (28) into Eq. (10), the electrical power per unit length is given now by

$$
P_{E}^{\beta}=\frac{8 R_{L} \zeta_{E}^{2} I_{C}^{2} U^{*}\left(1-\beta^{2}\right)}{3 a_{3}}\left(4 m^{*} \zeta_{T}-a_{1} U^{*}-4 m^{*} \zeta_{E} \beta^{2}\right),
$$

(a)

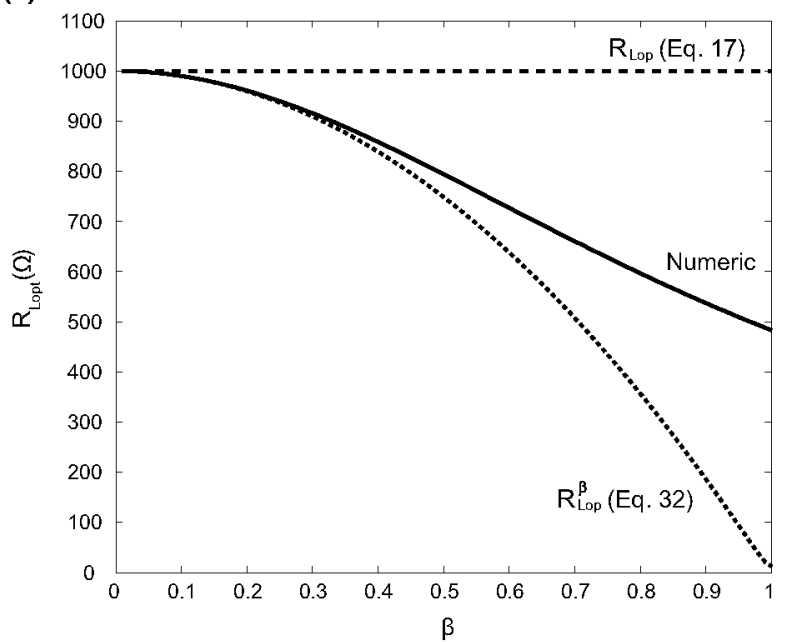

(b)

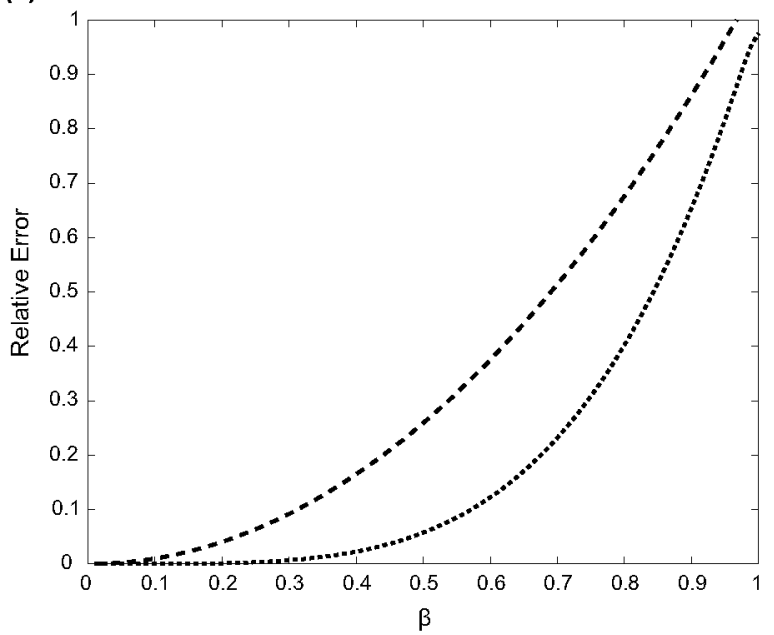

Fig. 5. (a) Optimal electrical load resistance variation with $\beta$ computed numerically (solid line) and given by analytic approximation (Eq. (32)). The optimal value predicted when inductance effects are not considered (Eq. (17)) is also shown (dashed line). (b) Relative error variation with $\beta$ for the analytic approximation when inductance effects are not considered (dashed line) and for the analytic approximation when inductance effects are considered (dotted line). $U^{*}=14.94$ and parameters listed in Table 1 . 
and the efficiency by

$$
\eta_{E}^{\beta}=\frac{16 R_{L} \zeta_{E}^{2} I_{C}^{2}\left(1-\beta^{2}\right)}{3 a_{3} \rho D \omega_{n}^{3} D^{3} U^{* 2}}\left(4 m^{*} \zeta_{T}-a_{1} U^{*}-4 m^{*} \zeta_{E} \beta^{2}\right)
$$

where, as said, $\beta^{3}$ terms have been neglected.

\subsubsection{Optimal electrical load resistance}

The optimal electrical load can be obtained by differentiating Eq. (30) with respect to $R_{L}$ and equating to zero; this leads to

$$
R_{\text {Lop }}=\frac{p}{q}\left(1-\beta^{2}\right)+\left(\left(\frac{p}{q}\right)^{2}\left(1-\beta^{2}\right)^{2}+R_{C}\left(R_{C}-\frac{p}{q}\left(1-\beta^{2}\right)\right)\right)^{1 / 2},
$$

which is valid up to order $\beta^{3}$. Note that Eq. (32) coincides with the result given in the analysis carried out for low frequency oscillations (Eq. (17)) when $\beta=0$. Fig. 5 shows a comparison of this result with numerical resolution of the complete system in order to quantify the validity of the assumptions made. To this end, an optimization method was employed to maximize the efficiency given by Eqs. (6) and (7) together with a fourth order Runge-Kutta scheme to numerically solve Eqs. (4a) and (4b). It has been considered to be the same parameters that were presented earlier in a practical example (Section 3.1.3, Table 1) and $U^{*}$ was fixed at a value of 14.93 (note that the optimal $R_{L}$ predicted was $1000 \mathrm{Ohms}$ in that case for $\beta=0$, see Fig. 4(a)), and $\beta$ was varied between 0 and 1 .

As it can be seen from Fig. 5(a) the $R_{\text {Lopt }}^{\beta=0}$ given for the simplified analysis where $\beta$ was neglected (dashed line) is a good approach up to values of beta around 0.1 where deviations between $R_{\text {Lopt }}^{\beta=0}$ and the numeric computed value (solid line) is around one per cent. Note that such an approximation leads to values of the electrical load higher than the required ones. The analytic prediction for $R_{\text {lopt }}^{\beta}$ where the inductance effect is considered by a second order perturbation approach (Eq. (32)), dotted line, is valid until $\beta$ values of 0.3 with deviations lower than one per cent with respect to the numerical computed value. As a comparison, for $\beta=0.3$ the relative error considering $R_{\text {Lopt }}^{\beta=0}$ would be around 10 per cent. For completeness, Fig. 5(b) shows the relative error for $R_{\text {Lopt }}^{\beta=0}$ and $R_{\text {Lopt }}^{\beta=0}$ as a function of $\beta$. As it can be seen, $R_{\text {Lopt }}^{\beta}$ is a valid approximation for $\beta$ values up to 0.6 (relative error less that 10 per cent).

\section{Concluding remarks}

An electro-fluid-elastic model has been presented to describe in a simplified way the electromagnetic energy harvesting from a flowing fluid taking advantage of Transverse Galloping oscillations. Two different levels of simplification have been made depending on the comparison between the characteristic electrical and mechanical timescales. In both cases, the effect of the electrical resistance load applied at the electromagnet on the energy harvested has been discussed analytically.

When the characteristic mechanical timescale is very large compared with the characteristic electrical time the inductance of the electromagnet can be neglected and the dynamical effect of the electromagnet in the galloping prism is just to introduce a damping force term. When the characteristic timescales are not so different the inductance needs to be considered since it introduces an additional stiffness term as well as a negative damping term into the dynamics on the galloping prism.

For fixed geometry and mechanical parameters, it has been found that there exists an optimal electrical resistance load for each fluid velocity of the incoming flow. When the optimal electrical resistance load is optimally fixed at each reduced velocity, the efficiency dependence with the reduced velocity is weakened in the sense that once the high level of efficiency is achieved it remains high over a large range of reduced velocities (its behavior is much more broadband than the case of a fixed value of the electrical resistance load). This is a remarkable result from the practical side and can be helpful to design control laws that maximize energy harvesting for changing flow velocity conditions. The importance of this is further highlighted by considering that it seems to be easier to tune the load impedance than physically altering the stiffness of the elastic support of the galloping prism. In addition, with respect to the optimal electrical load resistance, it has been shown that the inductance effects (that is, $\beta$ ) makes its value lower than for the case where $\beta=0$.

Finally, it should be noted that the work presented here is of a qualitative theoretical nature. That is, we aim to come up with a description of the system's basic behavior, based on a series of idealized hypothesis that reproduces generic trends, showing that there exists a link between the optimal electrical load resistance and the flow speed, but that may be too simplistic to reproduce its detailed engineering performance. This is the reason why, for example, as a first step, we have chosen to focus on the effect of fluid force related nonlinearities while keeping a simple linear modelling for other subsystems (mechanical, electrical, etc.). It is clear that these other nonlinearities may be important and they will be dealt with them in a future article but we still believe that our step-by-step approach may be useful to understand the overall system's behavior. Notwithstanding the fact that even if a full theoretical nonlinear model is developed and solved for all different parts, an actual experimental prototype should be manufactured and tested if the focus is placed on the engineering performance. 


\section{Acknowledgements}

This research has been funded by the international company REPSOL SA, through the REPSOL-INSPIRE program and under contract P120130288. The authors also want to acknowledge the anonymous reviewers who have helped us to improve the quality of the manuscript with their comments.

\section{References}

Abdelkefi, A., Hajj, M.R., Nayfeh, A.H., 2013. Piezoelectric energy harvesting from transverse galloping of bluff bodies. Smart Materials and Structures 22 (1), 015014.

Abdelkefi, A., Hajj, M.R., Nayfeh, A.H., 2012. Power harvesting from transverse galloping of square cylinder. Nonlinear Dynamics 70 (2), $1355-1363$.

Allen, J.J., Smits, S.J., 2001. Energy harvesting eel. Journal of Fluids and Structures 15, 629-640.

Barrero-Gil, A., Sanz-Andres, A., Alonso, G., 2010. Energy Harvesting from Transverse Galloping. Journal of Sound and Vibration 329 (14), $2873-2883$.

Barrero-Gil, A., Pindado, S., Avila, S., 2012. Extracting energy from Vortex-Induced Vibrations: a parametric study. Applied Mathematical Modelling 36 (7), $3153-3160$.

Bernitsas, M.M., Raghavan, K., Ben-Simon, Y., Garcia, E.M.H., 2008. VIVACE (Vortex Induced Vibration Aquatic Clean Energy): a new concept in generation of clean and renewable energy from fluid. Journal of Offshore Mechanics and Arctic Engineering, ASME Transactions 130 (4). $041101-15$.

Blevins, R., 1990. Flow-Induced Vibration. Van Nostrand Reinhold, New York.

De Broe, A.M., Drouilhet, S., Gevorgian, V., 1999. A peak power tracker for small wind turbines in battery charging applications. IEEE Transactions on Energy Conversion 14 (4), 1630-1635.

Doare, O., Michelin, S., 2011. Piezoelectric coupling in energy-harvesting fluttering flexible plates: linear stability analysis and conversion efficiency. Journal of Fluids and Structures 27 (8), 1357-1375.

El-hami, M., Glynne-Jones, P., White, N.M., Hill, M., Beeby, S., James, E., Brown, A.D., Ross, J.N., 2001. Design and fabrication of a new vibration-based electromechanical power generator. Sensors and Actuators A: Physical 92 (1173), 335-342.

Grouthier, C., Michelin, S., De Langre, E., 2013. Energy Harvesting by Vortex-Induced Vibrations in Slender Structures. Paper No. OMAE2013-10241, http:// dx.doi.org/10.1115/OMAE2013-10241.

Jung, H., Lee, S., 2011. The experimental validation of a new energy harvesting system based on the wake galloping phenomenon. Smart Materials and Structures 20, 055022 .

Koutroulis, E., Kalaitzakis, K., 2006. Design of a maximum power tracking system for wind-energy-conversion applications. IEEE Transactions on Industrial Electronics $53(2), 486-494$

Lee, J.H., Xiros, N., Bemitsas, M.M., 2011. Virtual damper-spring system for VIV experiments and hydrokinetic energy conversion. Ocean Engineering 38 (5), $732-747$.

Moor, G.D., Beukes, H.J., 2004. Maximum power point trackers for wind turbines. In: 35th Annual IEEE Power Electronics Specialists Conference, pp. 20442049.

Naudascher E., Rockwell D, 1994. Flow-Induced Vibrations: An Engineering Guide. A.A. Balkema, Rotterdam

Parkinson, G., 1989. Phenomena and modelling of flow-induced vibrations of bluff bodies. Progress in Aerospace Sciences 26 (2), $169-224$.

Païdoussis, M.P., Stuart, J.P., De Langre, E., 2011. Fluid-Structure Interactions. Cambridge University Press, New York.

Sanchez-Sanz, M., Fernandez, B., Velazquez, A., 2009. Energy-harvesting micro-resonator based on the forces generated by the Karman Street around a rectangular prism. Journal of Microelectromechanical systems 18, 449-457.

Raghavan, K., 2007. Energy Extraction from a Steady Flow using Vortex Induced Vibration (Thesis dissertation). University of Michigan.

Rizzoni, G., Hartley, T.T., 2000. Principles and Applications of Electrical Engineering. Mcgraw Hill, New York.

Sirohi, J., Mahadik, R., 2011. Harvesting wind energy using a galloping piezoelectric beam. Journal of Vibration and Acoustics 134 (1), 011009.

Zhao, L., Tang, L., Yang, Y., 2012. Small wind energy harvesting from galloping using piezoelectric materials, In: ASME 2012 Conference on Smart Materials, Adaptive Structures and Intelligent Systems, http://dx.doi.org/10.1115/SMASIS2012-8212. 